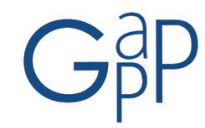

GESTIÓN Y ANÁLISIS DE POLÍTICAS PÚBLICAS, Nueva Época - N. 16, julio-diciembre 2016 - ISSN: $1989-8991$

DOI: http://dx.doi.org/10.24965/gapp.v0i16.10367

\author{
Martin Cutberto Vera Martínez \\ Universidad Autónoma de Baja California \\ martin.vera@uabc.edu.mx \\ David Rocha Romero \\ Universidad Autónoma de Baja California \\ drocha@uabc.edu.mx \\ Martha Cecilia Jaramillo Cardona \\ Universidad Autónoma de Baja California \\ martha.jaramillo@uabc.edu.mx
}

\title{
Medir a ciegas. Evaluación de desempeño en la Cruzada Nacional contra el Hambre
}

\section{Resumen}

El trabajo explora cómo el Programa de Comedores Comunitarios, integrante de un amplio esfuerzo para combatir la pobreza alimentaria en México, no está midiendo el impacto de los alimentos en los beneficiarios, sólo se sabe sobre la cobertura del programa. La investigación a través de metodología mixta (cualitativa y cuantitativa), entrevistas y encuestas, demostró que es casi nula la relación de las autoridades con los beneficiarios, no hay seguimiento de su salud y nutrición pues los indicadores previamente diseñados no lo contemplan. Esto se debe a una inadecuada aplicación de la Metodología del Marco Lógico que propone una relación estrecha entre autoridades y población objetivo, enmarcada por las propuestas de la Nueva Gestión Pública que empujan a los gobiernos a maximizar su eficiencia, su eficacia y desarrollar sistemas de medición de resultados.

Palabras clave

Pobreza alimentaria, medición, indicadores, Nueva Gestión Pública, México.

\section{Blindly measurement. Performance evaluation in the National Crusade against Hunger}

\begin{abstract}
The paper explores how the community kitchen program, part of a broad effort to combat food poverty in Mexico, is not measuring the impact of food on beneficiaries, we only know about the coverage of the program. Research through mixed methodology (qualitative and quantitative) interviews and surveys showed that the relation between the authorities and the beneficiaries was almost null, there is not a continuity of their health and nutrition because the indicators previously designed do not contemplate it. This is merely because there is an inadequate application of the Logic Framework Methodology marked by the proposals of the New Public Administration to push the government to maximize its efficiency, effectiveness and to develop systems for the measurement of results.
\end{abstract}

Keywords

Food poverty, measurement, indicators, New Public Management administration, Mexico.

* Los autores agradecen los comentarios de los dictaminadores que permitieron mejorar el artículo. 
In a Democracy, the government always acts so as to maximize the number of votes it will receive. In effect, it is an entrepreneur selling policies for votes instead of products for money.

Anthony Downs

\section{INTRODUCCIÓN}

En los últimos 30 años los gobiernos federales en México han diseñado e implementado políticas públicas para combatir la pobreza y la mala alimentación. De acuerdo con el Consejo Nacional de Evaluación de la Política de Desarrollo Social (CONEVAL) la carencia por acceso a la alimentación ${ }^{1}$ en México se incrementó de 20.789.646 personas en 2008 a 23.088.010 personas en 2014, de 21,7 por ciento a 23,4 por ciento de la población (CONEVAL, 2016). Baja California pasó de 428.600 personas en carencia de acceso a la alimentación en 2008 a 526.696 en 2010 (SEDESOL, 2012).

Así mismo, el índice de la Tendencia Laboral de la Pobreza², muestra que durante 2010 en zonas urbanas, el 45,5 por ciento de las personas no podía comprar la canasta alimentaria con sus ingresos, en las zonas rurales el porcentaje era de 59,98 por ciento. En 2014, en zonas urbanas subió al 52,39 por ciento y en zonas rurales a 62,70 por ciento. Baja California, Distrito Federal, Nuevo León, Quintana Roo y Sinaloa, en ese orden, son los estados con mayor índice de tendencia laboral de la pobreza en el país (Flores, 2015).

Ante tal reto, el gobierno federal creó en 2013 la Cruzada Nacional contra el Hambre (CNcH), encabezada por la Secretaria de Desarrollo Social (SEDESOL), que tiene entre sus principales objetivos erradicar la pobreza alimentaria, relacionada con la carencia de una dieta adecuada que aporte los nutrientes necesarios. La población objetivo de la $\mathrm{CNcH}$ en Baja California, hasta 2014 era de 71, 371 personas ${ }^{3}$. A la par de crear mecanismos institucionales para combatir la pobreza alimentaria, surge la necesidad de evaluar los alcances de las acciones gubernamentales. Evaluación en el sentido de la emisión de un juicio acerca de una política que se lleva mediante procedimientos sistemáticos de recolección de información suficiente y relevante, y análisis e interpretación de la información a través de comparaciones respecto a parámetros definidos (Padrón, 1995:173; Matos, 2005: 365; Di Virgilio y Solano, 2012: 39).

El objetivo de la investigación es demostrar que no se sabe cómo se está nutriendo a los beneficiarios, como uno de los objetivos principales del PCC y del PNMsH. Debido a que existe una escasa comunicación entre autoridades y los beneficiarios del programa, y que la nutrición y salud de éstos, no es monitoreada. No es intención del trabajo hacer una evaluación del PCC, y decir si está funcionando o no, ni tampoco presentar en qué medida está contribuyendo en el combate a la pobreza alimentaria. De la misma manera, no se ha planteado aquí proponer nuevos indicadores de resultados, para tal motivo se tendría que hacer un análisis exhaustivo de la problemática y eso escapa a los alcances del trabajo.

Las preguntas de investigación que guían este trabajo son ¿se ha implementado adecuadamente la Metodología del Marco Lógico (MML) en el diseño y aplicación del PCC y si se mide el impacto de los alimentos en las personas, para saber si se está combatiendo adecuadamente la pobreza alimentaria? Éste programa forma parte de una estrategia de intervención social orientada a promover cambios (De Miguel, 2000: 2929). Nuestra hipótesis de trabajo es que no se ha implementado adecuadamente la MML y consecuentemente no se están midiendo los impactos de los alimentos en las personas. Por tal motivo se llevó acabo un estudio mixto, cualitativo y cuantitativo, a través de entrevistas y encuestas, para analizar el funcionamiento de los comedores. Ya que la información disponible, tanto gubernamental como del CONEVAL, mide el desempeño del programa por los alcances logrados en base a la población objetiva beneficiada.

1 Se considera tomando en cuenta que por falta de dinero o recursos, tanto las personas mayores como menores de 18 años que no tuvieron una alimentación variada, comieron menos de lo necesario, se les disminuyeron las cantidades servidas de comida, sintieron hambre pero no comieron o los que sólo hicieron una comida al día o los que dejaron de comer todo el día.

2 Muestra la tendencia del porcentaje de personas que no pueden adquirir la canasta alimentaria con el ingreso labora. Si el índice sube, significa que aumenta el porcentaje de personas que no pueden comprar una canasta alimentaria con su ingreso laboral, CONEVAL. http:// www.coneval.org.mx/Medicion/EDP/Paginas/Evolucion-de-las-dimensiones-de-la-pobreza-1990-2014-.aspx. Consulta: 20 de mayo 2015.

3 http://www.sedesol.gob.mx/work/models/SEDESOL/Cruzada/Seleccion_Municipios_de_la_Segunda_Etapa_de_la_CNCH.pdf.COnsulta:10 de abril de 2015 . 
Se toma en cuenta la experiencia de tres comedores comunitarios en polígonos marginados de la ciudad de Tijuana, Baja California, México. Estos comedores como programa federal, conforman una actividad dentro del objetivo número 1 del PNMsH, que dice: «cero hambre a partir de una alimentación y nutrición adecuada de las personas en pobreza multidimensional extrema y carencia de acceso a la alimentación».

El objetivo del PNMsH, como del PCC, como se verá más adelante, no hacen distinción entre el subsidio de alimentos e intervenciones directas sobre la nutrición dirigida a grupos vulnerables, las cuales son más complejas en su ejecución y sus efectos muchas veces no son los esperados (Barquera, Rivera-Dommarco y Gasca-García, 2001: 469). Consecuentemente, los indicadores del programa tienen que ver con la parte de los subsidios y no con las intervenciones directas sobre la nutrición.

El trabajo se divide en cuatro partes, la primera presenta la teoría de la Nueva Gestión pública (NGP) que enmarca la razón de la MML y los antecedentes de la evaluación en México, seguida de la metodología mixta utilizada en la investigación, que consistió en entrevistas a funcionarios públicos, jóvenes coordinadores, quienes enlazan a las autoridades con los integrantes de los comités comunitarios, a miembros de estos comités, así como una encuesta a los beneficiarios. En la segunda parte se presenta la Matriz de Indicadores de Resultados (MIR) del PCC, enlazada a los objetivos del PNMsH. En la tercera parte se presentan los hallazgos, resultado del trabajo de campo y en la cuarta parte las conclusiones.

\section{TEORÍA}

En México y en el mundo existe un debate sobre cómo hacer más profesional, eficiente, eficaz, transparente y sometido a rendición de cuentas el gasto de los gobiernos para enfrentar los problemas públicos.

Tomando tendencias, principalmente anglosajonas, se planteó una nueva forma de administración pública, parecida a lo que hacen las organizaciones privadas (Arellano, 2004: 13). La lógica de la Nueva Gestión Pública (Aguilar, 2006), como cuerpo teórico, propone mejores prácticas para hacer gobiernos más eficaces, eficientes y abiertos a la participación de los ciudadanos.

Frente a esta nueva visión Barzelay (2003) expone el peligro que se tiene de tomar esta NGP como una tendencia, ya que su aceptación estimula la formulación de políticas por imitación antes que la resolución de problemas.

En este proceso surgen diversos enfoques que contribuyen a alimentar esta discusión donde se destacan Christopher Hood ${ }^{4}$ y Michael Jackson ${ }^{5}$ (1991) considerados los precursores del enfoque teorético de la NGP; junto con Peter Aucoin ${ }^{6}$ (1990). Sus trabajos representan la síntesis de la NGP. Varios autores sostienen sin embargo que la Nueva Gestión Pública, no es nueva sino es el resultado del avance en las ciencias administrativas (Gruening, 2001); por lo que lo que se podría delimitar en la NGP son un conjunto de características específicas tomadas de la academia, la política y la burocracia.

Aunado a estos postulados se han ido incorporando otras visiones como la creación de valor público de Moore, 1995, la gestión por procesos de Aguilar, 2006a, la legitimidad del estado de Cabrero, 2013, entre otros más que analizaron el fenómeno desde la investigación y la práctica.

«La administración pública elabora reglas, regulaciones y hechura de decisiones letárgica; mientras que el nuevo manejo público (la NGP) ostenta decisividad, imaginación y pasión por la actividad» (Vicher, 2007:165).

En este sentido, es importante recalcar la importancia del Marco Lógico como técnica, que dentro de la nueva gestión pública se presenta como herramienta orientada hacia el rendimiento y los resultados (Olías de lima, 2001:8). Como lo plantea Cejudo (C2013:17) la nueva gestión pública se orientada a flexibilizar estructuras y procesos en el sector público.

4 Christopher CROOPER HOOD, (1947), británico, profesor de la Universidad de Oxford, se especializo en el análisis de gobierno, políticas públicas y reformas del Estado. Entre sus obras más destacadas están: The Limits of Administration (1976), The Tools of Government (1983) y The Art of State (1998), entre otras.

5 PhD. Michael JACKSON, profesor emérito de la Universidad de Sidney, Australia, ha dedicado su investigación a la economía política, la administración pública y la ciencia política. Destacan sus obras sobre Jean Jacques Rousseau, Carl Smith y Georg Hegel.

6 Peter AucoIn (1943-2011), politólogo y profesor canadiense de la Universidad de Dalhousie, dedicó su carrera a la administración pública y las políticas de gobernanza. Fue asesor del Gobierno de Canadá para la reforma del Estado, y participó en administraciones locales en comisiones de reforma electoral, entre otros. 
Por lo tanto, surge la emergencia de un nuevo paradigma que permita acercar esas relaciones causales y abordarlas desde la Nueva Gestión Pública (NGP).

«Debe quedar claro, que el management es una orientación de un objeto de estudio mayor y del cual comparte su origen: la Administración Pública. Otro problema lo constituye la irrupción de ese paradigma autodenominado New Public Management (Nueva Gestión Pública), que es una reminiscencia añeja que incorpora las técnicas privadas a la administración pública, pero ahora se realiza mediante la vía de la economía neoclásica». (Sánchez, 2001, p. 77)

Este nuevo enfoque teórico surge tras la crisis del Estado de bienestar (keynesianismo) y la ola neoliberal (Thatcher y Reagan) de finales del siglo xx, que propendió a mejorar la ineficiencia burocrática que existía, como mecanismo para agilizar la dinámica teniendo como herramientas las nuevas tecnologías.

Por otra parte, Hood y Jackson sostienen que la NPG no debe ser considerada una teoría, sino una filosofía administrativa comúnmente aceptada. En su obra Administrative Argument (1991)7 sostiene que la emergente llamada NGP debe ser considerada como una doctrina, un tipo ideal de conducta en la esfera administrativa. Enfatizan que en tanto filosofía como argumento, la NGP toma referencias del cameralismo alemán del siglo XVIII, el utilitarismo británico del siglo XIX y el progresismo americano del siglo XX. (Barcelay, 2000).

En éste nuevo enfoque se formula la re-categorización del ciudadano como decisor, y no como un mero cliente consumidor; donde la interacción y el diálogo generan y retroalimentan el ejercicio de lo público. (Mariñez, 2013).

Lori Brainard y John Mcnutt (2010) afirman que éste nuevo rol enfatiza la creación de mayores alianzas entre ciudadanos, lo que a su vez genera compromisos estables en democracias. Al respecto afirman:

"Los administradores públicos crean oportunidades para el compromiso con los ciudadanos, utilizando esas oportunidades para educar, organizar y activar a éstos para promover y participar en la esfera pública en su propia representación. También significa que los administradores públicos se comprometerían con los ciudadanos a colaborar para identificar y definir los problemas, y crear y aplicar sus soluciones. Como parte de esto, las relaciones gobierno-ciudadanos se convertirían en más deliberativas y dialógicas antes que exclusivamente regulativas y basadas en la autoridad». (p. 841)

De acuerdo a Freddy Mariñez, este enfoque se presenta como una alternativa a lo que denomina la «administración pública tradicional» (old public administration) y frente a la lógica de la NGP (2013).

«Desde la perspectiva teorética, el Nuevo Servicio Público ofrece una importante y viable alternativa a los modelos gerenciales, tanto el convencional como el actualmente dominante. Esta es una alternativa que ha sido construida en base a las investigaciones teoréticas e innovaciones prácticas. El resultado es un modelo normativo, comparable con otros modelos. [... ] Las decisiones que toman los administradores públicos están marcadas fuertemente por los principios y argumentos en que se basen. Sí asumimos que la acción del gobierno es garantizar su propio interés entonces tomaremos un conjunto de medidas. Sí por otro lado, asumimos que la responsabilidad del gobierno está en promover la participación ciudadana, el debate y el interés público, entonces nosotros tomaremos un conjunto enteramente diferente de acciones». (Denhardt y Denhardt, 2000, p. 557).

Según Ostrom $(1973,157)$, es posible decir que la NGP apoya la participación de los ciudadanos una vez que el diseño del programa público ha pasado por los mecanismos de mercado.

En la NGP la racionalidad viene tanto de las reglas de mercado (el gobierno y la meritocracia orientados por los cuasi mercados y los resultados) como del empoderamiento de los ciudadanos (Nozick, 1974, 18-19; Gauthier, 1986, 444; Buchanan y Tullock, 1962).

Si, como defiende Rawls, la justicia debe venir antes que la «felicidad», la principal preocupación es el total desprecio de la NGP hacia el debate democrático, la deliberación y la discusión: la arena pública debe-

7 Publicada por DARMOUTH en Reino Unido (1991) y por BRooKflied en Estados Unidos (1992), es una obra que explica el surgimiento de la NGP en cuatro fases y que arguye que el estudio de casos comparados ha tendido a la internacionalización de las mismas normas de comportamiento de la gestión pública, lo que propició a organizaciones como la OCDE para proponerlas a sus países miembros. 
ría estar contenta con discutir asuntos técnicos y cómo el gobierno provee servicios, no en general cómo nuestros gobiernos son espacios de gobernanza, espacios para la discusión y la deliberación (Elster, 1998; Habermas, 1996).

Para Leeuw (1996, p. 92) la nueva gestión pública enfatiza en la aplicación de los conceptos de economía, eficiencia y eficacia en la organización gubernamental. Es decir, en primer lugar, el sector público reduce las diferencias respecto al sector privado en términos de personal, sistemas de remuneración y métodos de gestión; y, en segundo lugar, existe una disminución del volumen de reglas y procedimientos que articulan la actuación de gestión de los departamentos, sujetos a reglas uniformes para contratar e incurrir en costes (Dunleavy y Hood, 1994, p. 10).

Quizá la presencia en todas las teorías anteriormente analizadas de la necesidad de desarrollar y perfeccionar herramientas de control, especialmente orientadas a la evaluación de los logros o resultados obtenidos, se asocia en la literatura a la nueva gestión pública con la introducción de conceptos, prácticas y técnicas procedentes del sector privado (Fernández Rodríguez, 2000, p. 108), destinadas exclusivamente al control de resultados (Boden et al., 1998, p. 267 y Broadbent y Laughlin, 1998, p. 403), o se amplía a la introducción de mejoras en la gestión, mediante la desregulación, descentralización, y la introducción de competencia y transparencia en la rendición de cuentas (Coninck-Smith,1991; Ladner,1999; Montesinos, 1999).

Podríamos resumir que la Nueva Gestión Pública apremia la creación de una administración eficiente y eficaz, es decir, una administración que satisfaga las necesidades reales de los ciudadanos al menor coste posible, favoreciendo para ello la introducción de mecanismos de competencia que permitan la elección de los usuarios y a su vez promuevan el desarrollo de servicios de mayor calidad.

No obstante, la clasificación primaria utilizada para sintetizar estas actuaciones se basa en la ordenación secuencial lógica en el desarrollo de estas acciones tal y como se expone en el trabajo de Barea (1997).

Es por ello que el objetivo de las políticas de gestión pública exige la «desburocratización de las administraciones públicas» (García, 2007, p. 44), es decir, la simplificación de sus procedimientos internos, la horizontalidad en su creación y la transversalidad creativa en su desarrollo.

Con base en tales aportaciones, las actuaciones a desarrollar en el presente artículo centrarán su análisis en determinar cómo se han dado las relaciones con los ciudadanos y los programas públicos de alimentación.

\section{ANTECEDENTES DE LA EVALUACIÓN EN MÉXICO}

En 1995 se desarrollarían las primeras experiencias de evaluación del desempeño administrativo por medio de indicadores en el gasto público, lo que a la postre se convertiría en el Sistema de Evaluación de Desempeño (SED) (Arellano y Purón, 2004: 476).

A partir de 2006 se introducen obligaciones para evaluar los resultados de los programas sociales (Pérez-Jácome, 2009). Con la promulgación de la Ley General para el Desarrollo Social (LGDS), que hizo obligatoria la evaluación anual de los programas sociales, y de la Ley Federal de Presupuesto y Responsabilidad Hacendaria (LFPRH), la cual introduce el concepto de Presupuesto por Resultados (González y Velasco, 2014: 103). Se establecieron un conjunto de normas que modifican la forma de ejecutar los procesos de planeación, programación, aprobación, ejecución y evaluación en la administración pública federal (Ramos y Villalobos, 2012: 102). Por este medio se busca la efectividad y eficiencia del gasto público al vincular el funcionamiento de organizaciones del sector público a los resultados que producen. Utiliza información sistemática sobre el desempeño (indicadores, evaluaciones, costos de programas, etcétera) para establecer dicho vínculo (Maldonado, 2013: 14). La información sobre un producto se recaba a través de indicadores (Maldonado, 2013: 27). En la planeación del PBR la elaboración de indicadores debe dejar información clara sobre cómo evolucionan las decisiones gubernamentales y si se están cumpliendo cabalmente sus objetivos sin afectar intereses de otros sectores, por eso es muy importante su evaluación ${ }^{8}$. Para el 2004 se había creado el CONEVAL, organismo público descentralizado que evalúa la política social.

Por otro lado la LFPRH en 2006, asocia la evaluación de la política social a la construcción de un Sistema de Evaluación de Desempeño (SED), el instrumento operativo para la implementación del PBR (Acosta, 2011: 124),

8 Entendiendo por evaluación «La estimación sistemática y objetiva de un proyecto, programa o política en curso y de su diseño, implementación y resultados. El propósito es determinar la relevancia y el grado de cumplimiento de los objetivos, así como de la eficiencia, efectividad, impacto y sostenibilidad del desarrollo. Una evaluación debe proporcionar información creíble y útil que permita la incorporación de lecciones aprendidas al proceso de toma de decisiones. Así mismo la evaluación se refiere al proceso para determinar el valor o importancia de una actividad, política o programa; es una valoración lo más sistemática y objetiva posible de una intervención de desarrollo planificada, en curso o finalizada» (MALDONADO, 2013: 24). 
que se conforma de diversos procesos que permiten: i) evaluar los resultados de los programas federales; ii) retroalimentar a los administradores de dichos programas y iii) mejorar la calidad de información que se entrega al Congreso y a la sociedad.

Este mecanismo descansa en un Sistema de Indicadores de Desempeño, administrado en los hechos por la Secretaría de Hacienda y Crédito Público (SHCP). Como resultado, las dependencias y entidades deben elaborar la MIR (matriz de Indicadores para Resultados) de cada programa federal de acuerdo con la MML; La MIR formará parte del SED para coadyuvar en la eficiencia, economía, eficacia y calidad de la administración pública federal y en el impacto social del gasto público federal.

La MML es una herramienta para facilitar el proceso de conceptualización, diseño, ejecución y evaluación de proyectos. Presenta de forma sistemática y lógica los objetivos de un programa (Vera, 2015), contempla y facilita la participación de los involucrados en un problema público, particularmente en la elaboración de la MIR, ver esquema 1.

\section{ESQUEMA 1. METODOLOGÍA DEL MARCO LÓGICO. CONSENSOS Y ACUERDOS}

\section{Consensos y Acuerdos}

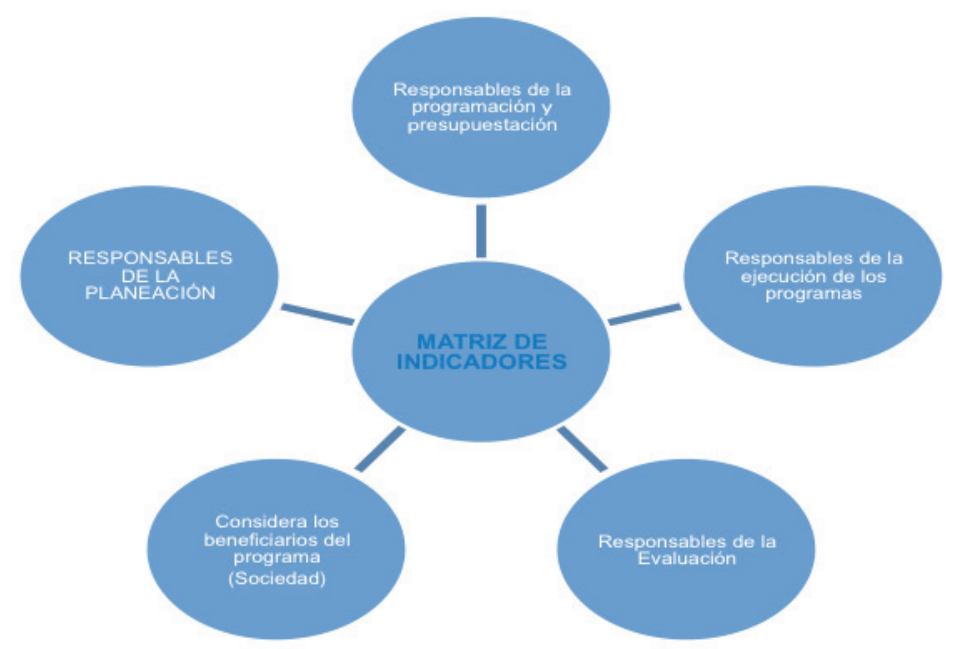

Principales involucrados en la elaboración de la MML.

Fuente. Vera MARTínez, Martín, 2015, Diseño y operación de indicadores de Evaluación en la AP, Manual de curso impartido con el auspicio de la Auditoría Superior de la Federación a funcionarios públicos.

La MML permite tener una visión panorámica de los problemas públicos que se presentan como fenómenos sociales interconectados y desarrollar hipótesis de causa-efecto, del tipo «si sucede A, entonces esto causa B» (Aldunate y Córdoba, 2011: 21), es un proceso que debe seguirse para llegar a la MIR. No es un producto final que sólo puede elaborarse por expertos en lo más alto de la jerarquía burocrática. No funciona sólo si se declara en documentos oficiales y en la práctica no se aplica, particularmente en el sentido de la comunicación entre las partes involucradas. Por medio del análisis de involucrados, análisis del problema, análisis de objetivos e identificación de alternativas de solución al problema, se identifican problemas y se dan alternativas de solución (Ortegón, Pacheco y Prieto, 2005). El análisis del problema y el análisis de objetivos ayudan a la construcción de indicadores de desempeño. El primero permite: 1) analizar e identificar lo que se considere como problemas principales, 2) establecer el problema central, aplicando criterios de prioridad y selectividad, 3) definir los efectos más importantes del problema en cuestión, 4) anotar las causas del problema central detectado, qué elementos están o podrían estar provocando el problema, 5) una vez que tanto el problema central como las causas y los efectos están identificados, se construye un árbol de problemas (el árbol es una imagen completa de la situación negativa existente), y 6) revisar la validez e integridad del árbol dibujado, asegurarse que las causas representen causas y los efectos representen efectos, que el problema central esté correctamente definido y que las relaciones (causales) estén correctamente expresadas.

El producto de la MML es la MIR, cuyos propósitos son establecer los objetivos y resultados esperados, definir los indicadores estratégicos para medir los resultados, definir los medios que permitan verificarlos, describir los bienes y servicios que entrega el programa a la sociedad (Vera, 2015). 
En lo referente a los indicadores, que son el resultado de poner en operación los objetivos a alcanzar (Di Virgilio y Solano, 2012:47), se requiere que consideren criterios de calidad, cantidad y tiempo para que generen información adecuada, así que se debe considerar que sean específicos, realizables, medibles, relevantes, enmarcados en el tiempo e independientes (Ortegón, Pacheco y Prieto, 2005: 34). Los indicadores de resultados deberán ser una expresión cuantitativa o cualitativa que permita, de una manera sencilla y fiable medir logros, reflejar los cambios vinculados con las acciones de los programas, monitorear y evaluar sus resultados (Acosta, 201: 137).

Además de generar indicadores que midan el cumplimiento de los objetivos de forma cuantitativa, indicadores de eficacia, como los porcentajes de población atendida por los programas sociales, es necesario proponer indicadores de calidad, de eficiencia, que evalúan atributos respecto a normas o satisfacción de los beneficiarios (Ramos y Villalobos, 2012: 119-121), debido a que los programas sociales precisamente están orientados a mejorar las condiciones de vida de la población objetivo.

En el caso de los programas alimenticios, ello equivale a preguntar ¿cómo están repercutiendo esos alimentos en la salud de las personas?

\section{METODOLOGÍA}

La investigación no hizo una evaluación del desempeño o del impacto, considerando los indicadores planteados por el PCC, sobre la población objetivo. Por el contrario, se efectúo trabajo de campo exploratorio para comprender cómo funciona el PCC en Tijuana, se llevó a cabo una investigación mixta, cuantitativa (encuestas) y cualitativa (entrevistas). Esta investigación mixta permite una investigación comprensiva del problema y es adecuada para abordar a grupos marginados, como los pobres (Creswell, 2003: 26). Se realizó una encuesta, no aleatoria, a 95 beneficiarios en tres comedores comunitarios, utilizando el denominado muestreo por cuotas, donde se determina una cantidad de individuos de una población para que sean miembros de la muestra, no habiendo un procedimiento especial, el criterio es arbitrario, eligiendo sujetos que comparten ciertas características, según la definición del estudio (Castañeda, 2011: 117). Con el propósito de saber si las autoridades se han acercado para dar seguimiento a su alimentación y salud nutrimental, se utilizaron sólo algunas de las preguntas aplicadas en la encuesta, ver anexo 1.

Estos tres comedores tienen similares características al resto de los comedores en Tijuana, de acuerdo a los criterios que ha desarrollado la SEDESOL para abrir tales establecimientos, considerando las condiciones socioeconómicas de las personas en polígonos marginados de la ciudad. Lo que interesa de los beneficiarios a la investigación, es conocer su relación con las autoridades. Se omitió hacer más encuestas en otros comedores, ya que se conoció de parte de 8 promotores, que tenían relación por lo menos con 25 comedores (cada promotor atiende en promedio a 3), que la situación de distanciamiento entre población y autoridades era la misma.

Por otro lado, se entrevistaron a funcionarios públicos de SEDESOL, así como a promotores, jóvenes que son los encargados de la logística del comedor, monitoreando su funcionamiento y coordinando la relación entre los comités comunitarios (grupo de personas de la localidad que se organizaron para dar de alta el comedor) y las autoridades. Las entrevistas tuvieron como eje preguntas sobre el desarrollo e implementación de la MML, particularmente en lo referente a la vinculación entre autoridades y beneficiarios. Estas actividades se llevaron acabo de febrero a agosto de 2015.

\section{EL PROGRAMA DE COMEDORES COMUNITARIOS}

La mala alimentación es causa de muchos problemas de salud que se convierten en problemas sociales, como la baja productividad provocada por el sobrepeso o el bajo aprovechamiento escolar, problemas que el Estado tiene la necesidad de atender. $\mathrm{La} \mathrm{CNcH}$ visualiza un objetivo, combatir la pobreza alimentaria, y así enfrentar diversos fenómenos sociales, véase esquema 2. La Cruzada inició formalmente el 22 de enero de 2013 con la publicación en el Diario Oficial de la Federación del Decreto por el que se instaura el Sistema Nacional para la CNcH. El 30 de abril de 2014, se publicó en el Diario Oficial de la Federación el PNMsH el cual constituye el instrumento de planeación oficial de la estrategia.

En el siguiente árbol de problemas, elaborado por SEDESOL, se observan diversos fenómenos que contribuyen a la baja ingesta de energía y nutrientes, y esto es causa de importantes problemas, como el bajo aprovechamiento escolar. 
ESQUEMA 2. ÁRBOL DE PROBLEMAS PNMSH

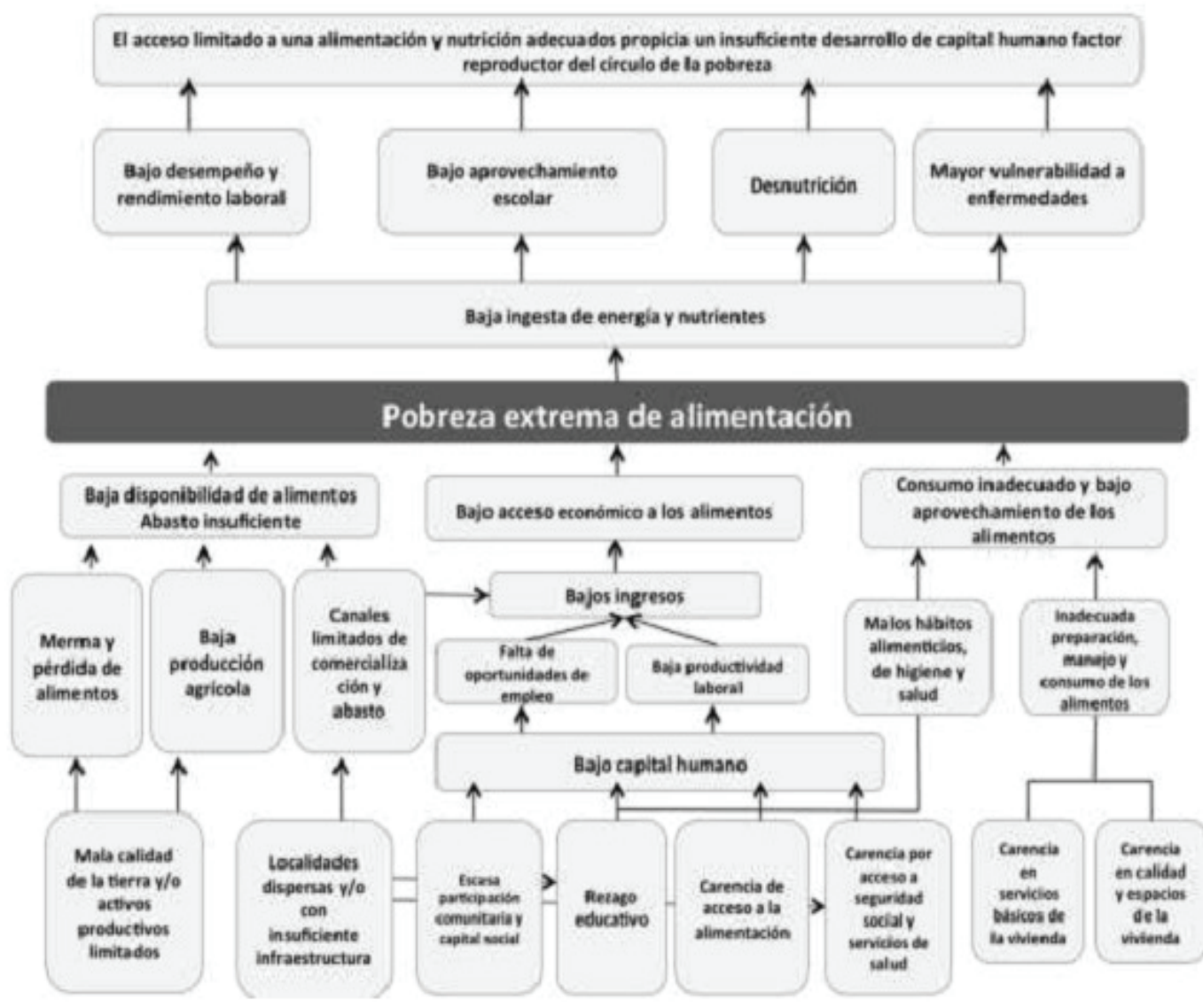

Fuente: Sin Hambre Cruzada Nacional. Elementos técnicos de diseño. Planeación e instrumentación del Programa Nacional México sin hambre. Julio 2014, SEDESOL. http://www.sedesol.gob.mx/work/models/SEDESOL/Cruzada/Programa_Nacional_Meexico_Sin_Hambre_Elementos_Metodologicos.pdf. Consulta: 30 de marzo de 2015.

El PNMsH contiene seis objetivos (componentes), el primero es relativo a la alimentación y nutrición adecuada, esquema 3.

ESQUEMA 3. MATRIZ DE MARCO LÓGICO PARA EL PROGRAMA NACIONAL MÉXICO SIN HAMBRE (PNMSH) 2014-2018

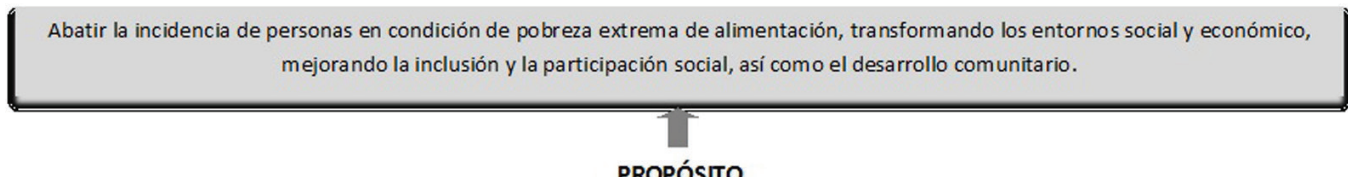

PROPÓsTOO

7.01 millones de personas superan la condición de pobreza extrema de alimentación.

\section{COMPONENTE 1}

Cero hambre a partir de una alimentación y nutrición adecuada de las personas en pobreza multidimensional extrema y carencia de acceso a la alimentación.

\section{COMPONENTE 4}

Minimizar las pérdidas post-cosecha y de alimentos durante su almacenamiento,

transportación, distribución y comercialización.

\section{COMPONENTE 2}

Disminuir la desnutrición infantil aguda crónica, y mejorar los indicadores de peso y talla de la niñez.

COMPONENTE 5

Promover el desarrollo económico y el empleo en las zonas de mayor

concentración de pobreza extrema de alimentación.

\section{COMPONENTE 3}

Aumentar la producción de alimentos y el ingreso de los campesinos y pequeños productores agricolas.

COMPONENTE 6

Promover la participación comunitaria para la erradicación del hambre.

Fuente: Sin Hambre Cruzada Nacional. Elementos técnicos de diseño. Planeación e instrumentación del Programa Nacional México sin hambre. Julio 2014. SEDESOL. http://www.sedesol.gob.mx/work/models/SEDESOL/Cruzada/Programa_Nacional_Meexico_Sin_Hambre_Elementos_Metodologicos.pdf. Consulta: 30 de marzo de 2015. 
El PNMsH define a la desnutrición como el resultado de una reducida ingestión alimentaria en un periodo prolongado de tiempo y/o de la absorción deficiente de los nutrientes consumidos. Generalmente asociada a una carencia de energía (o proteínas y energía), aunque también puede estar relacionada con carencia de vitaminas y minerales. La pobreza es considerada una causa de la malnutrición (obesidad y desnutrición). En México la metodología para la medición multidimensional de la pobreza desarrollada por el CONEVAL propone que existen dos referentes importantes relacionados con el acceso a los alimentos: el primero, la línea de bienestar mínima es un indicador de posibilidad económica de acceso que permite identificar a la población que, aun al hacer uso de todo su ingreso en la compra de alimentos, no puede adquirir lo indispensable para tener una nutrición adecuada. El segundo es la carencia de acceso a alimentación, que se construyó con base en la definición de seguridad alimentaria de la Organización de las Naciones Unidas para la Alimentación y la Agricultura (FAO por sus siglas en Inglés, 2006).

La Encuesta Nacional de Salud y Nutrición (ENSANUT), que utiliza índices antropométricos construidos a partir de mediciones de peso, talla y edad (Morales-Rúan, 2013: 201), arrojó datos de los beneficiarios de programas gubernamentales orientados a mejorar nutrición de los más pobres en comparación con el resto de la población, cuadro 1. La encuesta se levantó en 1988, 1999, 2006 y 2012 y demuestra que no es suficiente con dar alimentos cuantitativamente.

CUADRO 1. PREVALENCIA DE DESNUTRICIÓN EN MENORES DE 5 AÑos DE EDAD EN 1988, 1999, 2006 Y 2012. MÉXICO ENSANUT 2012

\begin{tabular}{|c|c|c|c|}
\hline \multicolumn{4}{|c|}{ Promedio nacional } \\
\hline & Bajo peso & Baja talla & Emaciación \\
\hline 1988 & 10,8 & 26,9 & 6,2 \\
\hline 1999 & 5,6 & 21,5 & 2,1 \\
\hline 2006 & 3,4 & 15,4 & 2 \\
\hline 2012 & 2,8 & 13,6 & 1,6 \\
\hline \multicolumn{4}{|c|}{ Promedio población oportunidades } \\
\hline 2012 & 4,3 & 21,4 & 1,9 \\
\hline
\end{tabular}

Fuente: Encuesta Nacional de Salud y Nutrición 2012. Estado de Nutrición, Anemia, Seguridad Alimentaria en la Población Mexicana http://ensanut.insp.mx/doctos/analiticos/Desnutricion.pdf. Consulta: 6 de abril 2015. Instituto Nacional de Salud Pública, Modulo de Oportunidades en ENSANUT 2011-2012. Tomado de Elementos Técnicos de Diseño, Planeación e Instrumentación del Programa Nacional México Sin Hambre, julio 2014. http://www.sedesol.gob.mx/work/models/SEDESOL/Cruzada/Programa_Nacional_Meexico_Sin_Hambre_Elementos_Metodologicos.pdf. Consulta: 6 de abril de 2015.

Este cuadro destaca que los beneficiados por el Programa Oportunidades en 2012, tienen peores condiciones relacionadas con la desnutrición que el promedio nacional, esto debería ser al revés. Los niños atendidos por el Programa deberían estar mejor nutridos y tener porcentajes más bajos de baja talla, bajo peso y emaciación. El Programa les da alimentos pero no necesariamente los nutre.

El cuadro 2 se presenta el objetivo número uno de la $\mathrm{CNcH}$, así como sus componentes, actividades e indicadores, contenidos en la MIR. Se observa a los comedores comunitarios como una actividad que contribuye con el indicador de disminuir el porcentaje de personas en pobreza extrema de alimentación.

Como se observa, es un indicador que mide el avance de 14 actividades, una de ellas son los comedores comunitarios. SEDESOL se refiere a ellos como «el espacio propicio para consumir alimentos nutritivos con base en dietas balanceadas, incluyendo ingredientes tradicionales y naturales de la región» (SEDESOL, 2013). El problema central que identifica el árbol de problemas de PCC es el «acceso insuficiente a alimentos variados y de calidad en los territorios urbanos y rurales de las Zonas de Atención Prioritaria».

El Objetivo general del PPC es «desarrollar y consolidar una estrategia de atención alimentaria y nutricional y, con ello, contribuir a abatir la carencia por acceso a la alimentación». En el Estado de Baja California había hasta abril de 2015, 183 comedores en sus 5 municipios, 86 en Tijuana, 49 en Ensenada, 38 en Mexicali, 5 en Rosarito y 5 en Tecate. 
Los objetivos específicos son: 1) Mejorar la situación nutricional de niños de 0 a 11 años de edad, mujeres en gestación y lactantes, personas con alguna discapacidad, adultos mayores de 65 años y aquellas personas que determine el comité comunitario, por su condición de pobreza multidimensional extrema y carencia de acceso a la alimentación; 2) Promover la inclusión social de la población atendida por los comedores, 3) Impulsar acciones de orientación nutricional entre la población atendida de los comedores, que sustente el desarrollo de una cultura alimentaria nutricional.

CUADRO 2. OBJETIVO 1, COMPONENTES, ACTIVIDADES E INDICADOR DE LA MIR DE LA PNMSH 2014

\begin{tabular}{|c|c|c|c|c|}
\hline Objetivo 1 & Componentes & & Actividades & Indicador \\
\hline $\begin{array}{l}\text { Cero hambre } \\
\text { a partir de una } \\
\text { alimentación y } \\
\text { nutrición adecuada } \\
\text { de las personas } \\
\text { en pobreza } \\
\text { multidimensional } \\
\text { extrema y carencia } \\
\text { de acceso a la } \\
\text { alimentación. }\end{array}$ & $\begin{array}{l}\text { C1.1 Incrementar } \\
\text { el acceso físico y } \\
\text { económico a alimentos } \\
\text { sanos y nutritivos. }\end{array}$ & f) & $\begin{array}{l}\text { Incrementar la cobertura de } \\
\text { los programas de transferencia } \\
\text { de ingreso a los hogares en } \\
\text { condiciones de pobreza extrema } \\
\text { de alimentación. } \\
\text { a.1. Incorporar nuevas familias al } \\
\text { padrón de oportunidades. } \\
\text { a.2. Incorporar nuevas familias al } \\
\text { padrón de PAL. } \\
\text { a.3. Incorporar nuevas familias al } \\
\text { padrón sin hambre. } \\
\text { Incentivar la adquisición de } \\
\text { alimentos básicos nutritivos } \\
\text { en los derechohabientes } \\
\text { de transferencia de apoyo } \\
\text { alimentario. } \\
\text { b.1. Promover el uso de la tarjeta } \\
\text { PAL sin hambre. } \\
\text { Incrementar los mecanismos } \\
\text { de atención a situaciones de } \\
\text { contingencia, ante eventos } \\
\text { coyunturales que vulneren el } \\
\text { derecho a la alimentación. } \\
\text { c.1. Apoyar con jornales del } \\
\text { programa de empleo } \\
\text { temporal. } \\
\text { Comedores comunitarios } \\
\text { y escuelas con asistencia } \\
\text { alimentaria. } \\
\text { d.1. Comedores comunitarios. } \\
\text { d.2. Escuelas de asistencia } \\
\text { alimentaria. } \\
\text { Promover el abasto de productos } \\
\text { alimenticios nutritivos en zona de } \\
\text { alta concentración de población } \\
\text { objetivo. } \\
\text { e.1 Tiendas en operación Diconsa. } \\
\text { Dotación y consumo de productos } \\
\text { alimenticios enriquecidos. } \\
\text { f.1. Dotar de leche fortificada a } \\
\text { fos nuevos beneficiarios. } \\
\text { f.2. Apertura nuevos centros de } \\
\text { acopio Liconsa. }\end{array}$ & $\begin{array}{l}\text { Disminuir el } \\
\text { porcentaje de } \\
\text { personas en } \\
\text { pobreza extrema } \\
\text { de alimentación } \\
\text { con ingreso } \\
\text { menor a la línea } \\
\text { de bienestar } \\
\text { mínimo. }\end{array}$ \\
\hline
\end{tabular}




\begin{tabular}{|c|c|c|c|c|}
\hline Objetivo 1 & Componentes & & Actividades & Indicador \\
\hline Mismo objetivo. & $\begin{array}{l}\text { C1.2 Aumentar la oferta } \\
\text { oportuna de alimentos } \\
\text { en territorios de mayor } \\
\text { concentración de } \\
\text { pobreza extrema de } \\
\text { alimentación. }\end{array}$ & a) & $\begin{array}{l}\text { Ampliar el sistema de abasto } \\
\text { social en zonas rurales y urbanas } \\
\text { de mayor concentración de } \\
\text { pobreza extrema de alimentación. } \\
\text { a.1. Apertura de nuevas tiendas } \\
\text { Diconsa y Tiendas de } \\
\text { operación Diconsa. } \\
\text { a.2. Atender localidades con } \\
\text { unidades móviles de Diconsa. } \\
\text { a.3. Centros de atención a } \\
\quad \text { derechohabientes del } \\
\text { esquema sin hambre. } \\
\text { a.4. Construir-instalar-apertura } \\
\text { nuevos comedores } \\
\text { comunitarios. } \\
\text { a.5. Comedores comunitarios en } \\
\text { operación. } \\
\text { Consolidar el sistema de abasto } \\
\text { social de leche para mejorar la } \\
\text { nutrición. } \\
\text { Mejorar los mecanismos de } \\
\text { protección contra riesgos que } \\
\text { puedan generar inseguridad } \\
\text { alimentaria: desastres, } \\
\text { emergencias, sequías, conflictos y } \\
\text { fluctuaciones económicas. }\end{array}$ & $\begin{array}{l}\text { Disminuir el } \\
\text { porcentaje de } \\
\text { personas en } \\
\text { pobreza extrema } \\
\text { de alimentación } \\
\text { con carencia } \\
\text { por acceso a la } \\
\text { alimentación } \\
\text { (7,01 millones de } \\
\text { personas, 100\%). }\end{array}$ \\
\hline
\end{tabular}

Fuente: Elementos Técnicos de Diseño, Planeación e Instrumentación del Programa Nacional México Sin Hambre, julio 2014. http://www. sedesol.gob.mx/work/models/SEDESOL/Cruzada/Programa_Nacional_Meexico_Sin_Hambre_Elementos_Metodologicos.pdf. Consulta: 6 de abril de 2015.

Los indicadores de resultados para cumplir con los objetivos anteriores, en teoría, se asocian al cumplimiento de los objetivos fundamentales de una política pública y deben medir lo verdaderamente esencial (Salcedo, 2011: 34). Los indicadores del PCC miden en términos cuantitativos el desarrollo del programa pero omiten la situación nutricional que es parte de sus objetivos específicos. Todos los medios de verificación de los indicadores tienen que ver con cuantificar el número de elementos del indicador. Por ejemplo, el porcentaje de comités comunitarios en los que participan mujeres, obtenidos del Registro de la Dirección General de Participación Social, cuadro 3.

CUADRO 3. INDICADORES DEL PROGRAMA DE COMEDORES COMUNITARIOS

\begin{tabular}{|l|}
\hline \multicolumn{1}{|c|}{ Nombre del indicador } \\
\hline Indicador del FIN. Porcentaje de la población con acceso a la alimentación. \\
\hline Indicador de PROPÓSITO Porcentaje de AGEBs intervenidas en el área de cobertura urbana del programa. \\
\hline Indicador de PROPÓSITO. Porcentaje de localidades rurales intervenidas en el área de cobertura del Programa. \\
\hline $\begin{array}{l}\text { Indicador de COMPONENTES. Porcentaje de comedores comunitarios instalados y en operación dentro del área } \\
\text { de cobertura del Programa. }\end{array}$ \\
\hline $\begin{array}{l}\text { Indicador de COMPONENTES. Porcentaje de comedores comunitarios solicitados e instalados dentro del área } \\
\text { de cobertura del Programa. }\end{array}$ \\
\hline $\begin{array}{l}\text { Indicador de COMPONENTES. Porcentaje de personas que participan en la preparación y ministración de } \\
\text { alimentos del comedor. }\end{array}$ \\
\hline
\end{tabular}




\begin{tabular}{|l|}
\hline \multicolumn{1}{|c|}{ Nombre del indicador } \\
\hline Indicador de COMPONENTES. Porcentaje de cocinas comunitarias con equipamiento instaladas. \\
\hline Indicador de COMPONENTES. Porcentaje de personas atendidas en los comedores comunitarios. \\
\hline Indicador de COMPONENTES. Promedio de mujeres atendidas en los comedores comunitarios. \\
\hline $\begin{array}{l}\text { Indicador de COMPONENTES. Porcentaje de municipios de la Cruzada contra el Hambre atendidos con al menos } \\
\text { un comedor comunitario. }\end{array}$ \\
\hline Indicador de COMPONENTES. Porcentaje de Comités Comunitarios constituidos. \\
\hline $\begin{array}{l}\text { Indicador de ACTIVIDADES. Porcentaje de proyectos de comedores comunitarios autorizados por la dirección } \\
\text { General de Participación Social. }\end{array}$ \\
\hline $\begin{array}{l}\text { Indicador de ACTIVIDADES. Porcentaje de Asambleas Comunitarias celebradas para la constitución de comités } \\
\text { comunitarios. }\end{array}$ \\
\hline Indicador de ACTIVIDADES. Porcentaje de comités comunitarios en los que participan mujeres. \\
\hline Indicador de ACTIVIDADES. Porcentaje de comités comunitarios liderados por mujeres. \\
\hline Indicador de ACTIVIDADES. Porcentaje de comedores comunitarios con supervisión y seguimiento. \\
\hline
\end{tabular}

Fuente: Secretaría de Desarrollo Social (SEDESOL). http://www.sedesol.gob.mx/work/models/SEDESOL/EvaluacionProgramasSociales/ MIR_2014/MIR_2014_PCC.pdf. Consulta: 19 de abril 2014.

Los indicadores antropométricos utilizados comúnmente en encuestas se derivan de mediciones de peso, estatura (o longitud) en infantes, niños, adolescentes y adultos (SEDESOL, 2012: 47) y contribuyen al seguimiento de la desnutrición ${ }^{9}$. Indicadores que hagan referencia a la ingesta alimentaria y a la utilización biológica de los alimentos, que permiten conocer el estado nutricional de cada persona, complementan a los indicadores de cobertura, que son parte del grupo de indicadores de eficacia.

\section{HALLAZGOS}

En 2014 el CONEVAL realizó la encuesta Monitoreo de Indicadores de Desarrollo Social en los 400 municipios iniciales de la Cruzada, teniendo como propósitos: 1) conocer las características sociodemográficas y socioeconómicas de la población que habita dentro de los 400 municipios que cubre en su etapa inicial la Cruzada, 2) caracterizar a esta población, municipios con alto porcentaje de población en pobreza extrema y pobreza extrema alimentaria y municipios con elevado volumen de población en pobreza extrema y pobreza extrema alimentaria, 3) delinear los perfiles de 5 de 400 municipios seleccionados y 4) conocer la cobertura de algunos programas sociales en esta población al inicio de la Cruzada (CONEVAL, 2014). En la información recabada en 2014, no se obtuvieron datos relacionados con la salud nutrimental de las personas, pero si se estableció como importante «tener indicadores de resultados relacionados con la población en pobreza extrema alimentaria que permita cuantificar su atención y darle seguimiento a sus resultados» (CONEVAL, 2014). La misma evaluación recomienda monitorear la evolución de la desnutrición y la anemia en aquellas poblaciones que continúan presentando las mayores prevalencias, como son los menores de 2 años, los adolescentes, las mujeres en edad fértil, los adultos mayores e indígenas, entre otras recomendaciones (SEDESOL, 2014).

Por otro lado, en la auditoría realizada por la Auditoria Superior de la Federación, se informó que en 50 de los programas incluidos en la PNMSH, no fue suficiente la información que reportaron para la mejora en el acceso de las personas a alimentos nutritivos (ASF, 2014). Tiene mucha relevancia lo anterior, pues la calidad de un programa social se mide por su compromiso en la resolución de los problemas sociales (De Miguel, 2000: 294)

Por otra parte, el Diagnóstico y Propuesta de Atención del Programa de Comedores Comunitario (SEDESOLb, 2014 ), no aborda la implementación de nuevos indicadores de calidad, como el de la medición antropométrica periódica, tampoco si existe una comunicación y coordinación entre agentes gubernamentales y los comités comunitarios para el desarrollo del programa.

9 El Instituto Nacional de Salud Pública cuantifica los índices antropométricos a través de ENSANUT y mostró que en 2012 en todo el país, 302 mil 279 menores de 5 años de edad presentaban bajo peso; 1 millón 467757 baja talla y 171 mil 982 emaciación. La desnutrición provoca anemia. http://www.sedesol.gob.mx/work/models/SEDESOL/Resource/2910/1/images/Diagnostico_2014_PCC.pdf. Consulta: 17 de julio de 2015. 
Los indicadores (cuadro 3) reflejan el alcance del programa medido en porcentaje de cobertura, y no el impacto de los alimentos en los beneficiarios. Así también, en el desarrollo del programa, la participación de los beneficiarios en la toma de decisiones y recopilación de información, es muy limitada; contrario a lo postulado por la teoría, donde se contempla y facilita la participación de los beneficiarios. Las decisiones que toman los comités se limitan a la elaboración de la comida, con los ingredientes que envía el gobierno o a quienes se les permite entrar a comer ${ }^{10}$. En este sentido, tanto los beneficiarios como los promotores de los comedores comentaron que no se ha dado, por lo menos en el último año, el acercamiento de alguna autoridad gubernamental, de cualquier dependencia que colabora en la $\mathrm{CNcH}$, como por ejemplo personal de la Secretaría de Salud o de la propia SEDESOL o alguna otra más, para monitorear la salud de los beneficiarios, en ningún sentido, a través de una encuesta, prueba médica, diagnóstico, etcétera. Esto en gran medida se debe a que «En los comedores no dejan pasar a gente de salubridad, sólo una señora de las organizadoras compró una báscula y ha pesado a los niños. No hemos visto ni escuchado nada sobre estudios clínicos o diagnósticos médicos hacia los beneficiarios. Incluso varios promotores se han enfermado de tifoidea, nosotros comemos en los comedores, no sabemos si se han enfermado por comer en los comedores, hay poco control de calidad. Hasta hace poco revisamos la caducidad de los productos. En algunos lugares hemos dejado de comer por la calidad y limpieza dudosa. Hay comedores de piso de tierra. Muchos comedores son compromisos políticos, que no son lugares aptos para abrir un comedor» ${ }^{11}$.

Por otro lado, la encuesta que se levantó, no aleatoria, en tres comedores comunitarios, encontró que ha sido casi nula la comunicación entre autoridades y beneficiarios. A pregunta expresa, ¿desde que come en el comedor, alguna autoridad se le ha acercado para vigilar su salud o talla-peso?, el 91 por ciento de los 90 beneficiarios que contestaron la pregunta, contestó negativamente. Esto a pesar que el 54,8 por ciento de los beneficiarios encuestados, acuden de 5 a 7 días a los comedores, de estos. El 37 por ciento, lo ha hecho durante 5 meses, es decir, han hecho durante un tiempo prolongado un uso constante de los comedores. El restante 45 por ciento acude a los comedores de 1 a 4 días a la semana. Ya que es muy frecuente la asistencia a los comedores, es factible la posibilidad de monitorear la salud alimentaria de los beneficiarios, si ese fuera un indicador.

Los mismos padres no tienen información clara de cómo estos alimentos benefician a sus hijos. 40 por ciento de los beneficiarios adultos, tienen hijos que acuden a los comedores, de estos 36,8 por ciento, creen que sus hijos han subido de peso, 39 por ciento creen que se han mantenido en su peso y 23 por ciento no saben. En promedio son 3 los hijos por padre o madre que acuden a los comedores. Del mismo modo, los padres no están ciertos de cómo los alimentos recibidos por sus hijos potencia sus capacidades. El 36 por ciento de los padres cuyos hijos acuden a los comedores dijeron que han mejorado sus calificaciones, 19 por ciento dijeron que siguen igual y 44 por ciento no saben si ha habido variaciones en su desempeño escolar. No hay información que ayude a ver como los alimentos repercuten en la población objetivo.

\section{CONCLUSIONES}

El trabajo de campo, mostró la escasa relación y comunicación entre autoridades y beneficiarios y esto propicia que no se este evaluando la nutrición de las personas que se alimentan por el PCC. El proporcionar alimentos no es suficiente para combatir los problemas relacionados con la pobreza alimentaria, pues no es sólo cuestión de cantidad sino de calidad. Por el momento no se sabe cómo los alimentos impactan en la salud de los beneficiarios.

El PNMsH en la etapa 2 de la evaluación de los resultados intermedios, tiene en cuenta un análisis de plan de cobertura a corto y mediano plazo, así como sus sistemas de información y los mecanismos de rendición de cuentas de las estrategias. En la etapa 4 de la evaluación de impacto se hará un análisis cuantitativo utilizando el Censo 2010 y el conteo poblacional 2015. Mientras tanto, miles de millones de pesos se han gastado y poco podemos decir sobre el avance de la salud nutrimental.

Mediciones antropométricas u otros tipos de mediciones sobre talla y peso, así como instrumentos que permitan ver como los alimentos repercuten en las personas, deben estar entre las acciones de las autoridades que pretenden mejorar la salud nutrimental de los beneficiarios de estos programas sociales. En un principio, un indicador que mida el impacto de la comida en las personas, es indispensable para saber si los recursos se están destinando adecuadamente, por ahora no se sabe si los alimentos están provocando algún efecto nutricional en los consumidores.

10 Entrevista a Cocinera en un comedor comunitario de Tijuana, febrero 2015.

11 Entrevista a promotoras de los comedores comunitarios, febrero 2015. 
Los procesos evaluativos se orientan a una de las tres funciones básicas: 1) la rendición de cuentas, la mejora de los programas y el incremento del conocimiento al respecto del mismo, con los propósitos de comprobar si los objetivos de los programas han sido alcanzados, 2) recabar opiniones fundamentadas sobre la valoración que hacen del programa las audiencias implicadas y 3) obtener información cualificada partir de la cual se puedan tomar decisiones sobre el futuro de un programa específico (De Miguel, 2000: 306). En el programa analizado no se cumple el criterio número dos, recabar información de las audiencias implicadas.

En el trabajo de campo en los comedores, se observó la falta de comunicación entre beneficiarios (comités comunitarios y comensales) y autoridades, provocando improvisación en la toma de decisiones. La voz de los comités comunitarios, que son las organizaciones vecinales que aterrizan el programa en los barrios, los encargados de elaborar los alimentos y ofrecerlos a los beneficiarios, no se ha tomado en cuenta, tanto en el proceso de la generación de indicadores como en el desarrollo del programa, como por ejemplo, ¿dónde son los lugares más adecuados para poner los comedores dentro del polígono de pobreza seleccionado?

A más del 90 por ciento de los beneficiarios (comensales) ninguna autoridad se le ha acercado para medir la evolución de su salud nutrimental, vigilando su talla y peso. Lo mismo sucede con los niños que acuden a los comedores. Esto a pesar del uso constante y prolongado de los comedores, medido en días de la semana y meses acudiendo a ellos. Tomando en cuenta la asistencia, hay buenas posibilidades de monitorear a las mismas personas.

Al parecer los indicadores del PCC fueron diseñados desde la óptica de los subsidios a los alimentos y no desde el punto de vista de las intervenciones directas sobre nutrición dirigida a grupos vulnerables, por tal motivo no hay indicadores que evalúen la nutrición de los beneficiarios.

Al mismo tiempo, la participación de los beneficiarios en los programas es deseable, tanto en la planeación, monitoreo y la evaluación (Barquera, Rivera-Dommarco y Gasca-García, 2001: 475).

Muchos indicadores de las políticas públicas en México se generan dependiendo de los intereses de los tomadores de decisiones bajo 3 criterios: a) La lógica de la política y la manipulación electoral: Objetivos de corto plazo que se midan bajo indicadores cuantitativos, teniendo en cuenta la competencia electoral y el abordar problemas de alto impacto. El calendario electoral es más corto y muchas veces no empata con los resultados de las políticas públicas, b) Abaratamiento de los costos de la gestión pública: Debido a una mayor austeridad, los tomadores de decisiones plantean indicadores que se nutren de información de fácil acceso, misma que, muchas veces ya se generó. En consecuencia no se plantean estrategias para crear nueva información, que serían más costosas. Se presentan buenos resultados con los datos ya existentes, generados por otras dependencias y no por la nueva política pública, c) El miedo al fracaso de sus acciones, por eso se crean indicadores favorables que dejan ver el «éxito de las administraciones", como dotar de desayunos escolares y el indicador es el número de desayunos, no el efecto que estos tienen en los niños. Entre más desayunos, mejor desempeño del gobierno. Hay falta de disposición por parte de los ejecutores para que se estudie la incidencia del programa (Nina, 2008: 454).

Sumado a esto hay una limitada cultura de la evaluación en la gestión pública, son muchos los funcionarios públicos que conservan el «síndrome de Blanca nieves»: la evaluación es correcta y objetiva y no hay objeciones mientras el espejo mágico diga que uno es la encarnación de la eficacia; pero cuando el espejo es objetivo y nos dice que no somos los mejores ni los más eficaces, entonces confrontamos lo dicho y se emprenden campañas de desprestigio contra los sistemas de evaluación (Salcedo, 2011, 37). En muchos casos, prevalece el Estado megalómano, él que puede resolver todo sin pedir ayuda a los demás.

Recientemente la SEDESOL anunció la utilización del Sistema de Focalización del Desarrollo, SIFODE ${ }^{12}$, donde se puede obtener información de diferentes instituciones y programas, y cruzar datos de beneficiarios del PCC y mediciones antropométricas que elaboren otras dependencias como la Secretaría de Salud y,así, plantear indicadores de calidad. Evolucionar a medir más que la cantidad, la calidad de las acciones gubernamentales. Esto se vuelve una necesidad imperante ante los escenarios de crisis e incremento de la pobreza. El PCC crecerá por los millones que buscarán alimentos nutritivos a bajo costo y se debe tener certeza que los miles de millones de pesos invertidos en éste programa, impactarán favorablemente a las poblaciones más vulnerables, de otra manera, se estará buscando el grial de la pobreza en la obscuridad.

12 Tiene como objetivo la focalización de participantes para la atención por parte de los programas sociales a través de la integración de información socioeconómica: La base de datos que contiene información social, económica y demográfica de los hogares, sus integrantes y las características de sus viviendas o de las comunidades u organizaciones, la cual se recolecta por los programas sociales y tiene como principal objetivo identificar potenciales usuarios. Lineamientos de Integración, Uso y Compartición de Información Socioeconómica del SIFODE. http://www.normateca.sedesol.gob.mx/work/models/NORMATECA/Normateca/1_Menu_Principal/2_Normas/2_Sustantivas/Lineamientos_SIFODE. pdf. Consulta: 20 de julio de 2015 . 


\section{BIBLIOGRAFÍA}

AucoIN, P. (1995), The New Public Management: Canada in Comparative Perspective, Montreal: IRPP.

ACOSTA, Félix, (2011), "Evaluando la política social y desempeño de gobierno en México: Tensiones y dificultades institucionales", en RAMOS, José María, José SOSA y Félix AcOSTA (coordinadores), La Evaluación de políticas públicas en México, El Colegio de la Frontera Norte, Instituto Nacional de Administración Pública, A. C.

AUDITORIA SUPERIOR DE LA FEDERACIÓN (ASF) (2014), Auditoria de Desempeño. En línea: http://www.asf.gob.mx/ Trans/Informes/IR2013i/Documentos/Auditorias/2013_0275_a.pdf. Consulta: 27 de abril de 2015.

Aguilar VILlanUeVA, Luis F. (2006), Gobernanza y gestión pública. México D. F.: Fondo de Cultura Económica.

ALDUNATE, Eduardo y Julio CóRDOBA (2011), Formulación de programas con la metodología del marco lógico, Serie Manuales, Instituto Latinoamericano y del Caribe de Planificación Económica y Social (ILPES), CEPAL, Naciones Unidas.

Arellano Gault, David (2004), "Nueva gestión pública: ¿dónde está lo nuevo? Bases para el debate de la reforma administrativa", en ARELLANO GAULt, David (coordinador), Más allá de la reinvención del gobierno: Fundamentos de la nueva gestión pública y presupuestos por resultados en América Latina. México D. F.: Miguel Ángel Porrúa, Centro de Investigación y Docencia Económica.

Arellano Gault, David y Gabriel PuRón (2004), "México. Reforma al sistema presupuestal: una reforma atrapada por las inercias", en ARELLANO GAULT, David (coordinador), Más allá de la reinvención del gobierno: Fundamentos de la nueva gestión pública y presupuestos por resultados en América Latina. México D. F.: Miguel Ángel Porrúa, Centro de Investigación y Docencia Económica.

AucoIN, P. (1995), The New Public Management: Canada in Comparative Perspective, Montreal: IRPP. DOI: 10.1017| So00842390000826X.

BARCELAY, M; y JACOBSEN, A. (2009), "Theorizing Implementation of Public Management Policy Reforms: A Case Study of Strategic Planning and Programming in the European Commission" en Governance: An International Journal of Policy, Administration, and Institutions, vol. 22, núm. 2, pp. 319-334. Massachusetts, US: Wiley.

BAREA, J. (1997): "Un Sector Público para el siglo XXI", Conferencia pronunciada en el Club Siglo XXI, Boletín AECA, núm. 42, pp. 3-15.

Barquera, Simón, Rivera-Dommarco, Juan y Gasca-García, Alejandra (2001), "Políticas y programas de alimentación y nutrición en México", Salud Pública de México, vol. 43, núm. 5, septiembre-octubre, 464-477. DOI: $10.1590 /$ S0036-36342001000500011.

BARZELAY, Michael (2003). La nueva gestión pública, México: Fondo de Cultura Económica.

Boden, R., GummetT, P., COX, D. y BARKer, K. (1998): "New Public Management and the Funding of Science and Technology Services To The UK Government", Accounting, Auditing and Account ability Journal, vol. 11, núm. 3, pp. 267-291.

BRoAdBENT, J. y LAUGHLIN, R. (1998): "Absorption and Absorbing Groups in Schools and GP Practices in the UK", Accounting, Auditing And Accountability Journal, vol. 11, núm. 3, pp. 403-434.

BUCHANAN, J. y G. TULLOCK (1962), Calculus of Consent, Ann Arbor, University of Michigan Press.

Cabrero MendozA, Enrique (2013). "Cambio en la administración pública" en Nueva Gestión Pública, CEJudo, Guillermo (comp.): Siglo Veintiuno Editores \& Biblioteca Básica de Administración Pública. Segunda reimpresión. México.

CASTAÑEDA, Juan (2011), Metodología de la investigación, segunda edición. México D. F.: McGraw-Hill.

CONINCK-SMITH, N. de (1991): "Restructuring for Efficiency in the Public Sector", McKinsey Quarterly, núm. 4, Pp. 133-150.

Consejo Nacional de EValuación de la Política de Desarrollo Social (CONEVAL) (2014). http://www.coneval. gob.mx/Informes/Evaluacion/IEPDS_2014/IEPDS_2014.pdf. Consulta: 21 de abril de 2014.

CRESWELL, J. W. (2003). Qualitative, cuantitative and mixed methods approaches, Qualitative Inquiry and Research Design: Choosing Among Five Approaches (Second Edit). USA, SAGE Publications, Inc. En línea: http://isites. harvard.edu/fs/docs/icb.topic1334586.files/2003_Creswell_A\%20Framework\%20for\%20Design.pdf. Consulta: 30 de Julio de 2016.

Consejo Nacional de EValuación de la Política de DesarRollo Social (CONEVAL) (2016), http://www.coneval. org.mx/Medicion/EDP/Paginas/Evolucion-de-las-dimensiones-de-la-pobreza-1990-2014-.aspx. Consulta: 21 de abril de 2016.

DE Miguel DíAz, Mario (2000), "La Evaluación de programas sociales: Fundamentos y enfoques teóricos”, Revista de Investigación Educativa, vol. 18, núm. 2: 289-317.

DenhardT, R; y DenHARDT, J. (2003), The New Public Service. Serving, not Steering, New York: Sharpe.

DI VIRGILIO, María Mercedes y SOLANO, Romina (2012), Monitoreo y evaluación de políticas, programas y proyectos sociales, Argentina, CIPPEC, Fondo de las Naciones Unidas para la Infancia (UNICEF). En línea: http://www. unicef.org/argentina/spanish/cippec_uni_monitoreo_evaluacion.pdf. Consulta: 1 de julio de 2015. 
Dunleavy, P. J. y Hood, C. (1994): “From Old Public Administration to New Public Management”, Public Money and Management, vol. 14, núm. 3, pp. 9-16.

ELSTER, Jon (1989), The Cement of Society, Cambridge, Cambridge University Press.

- (1992), Local Justice, Londres, R. Sage.

- (1997), Egonomics, Barcelona, Gedisa.

- (1998), Deliberative Democracy, Cambridge, Cambridge University Press.

Fernández Rodríguez, E. (2000), “La Nueva Gestión Pública: New Public Management”, Partida Doble, núm. 111, mayo.

GARcíA, J. (2010), "Prácticas y Políticas exitosas para promover la mejora regulatoria y el emprendimiento a nivel sub-nacional” [en línea], en Documentos de Trabajo OCDE sobre Gobernanza Pública, núm. 18, París: Publicaciones OCDE. Disponible en: http://www.oecd.org/gov/regulatory-policy/46003827.pdf. Consulta: 18 de enero de 2016.

GAUTHIER, D. (1986), Morals by Agreement, Oxford, Oxford University Press.

GONZÁLEZ GÓMEZ, Javier y Ernesto Velasco Sánchez (2014), “La Evolución del presupuesto basado en resultados en México, 2004-2012: transferencia e implementación de una política pública”, Revista del CLAD Reforma y Democracia, núm. 58, febrero: 101-134.

GrueninG, G. (2001), "Origin and theoretical basis of New Public Management", en International Public Management Journal, [en línea], núm. 4, pp.1-25. En Línea: http://eclass.uoa.gr/modules/document/ file.php/PSPA108/4NPM\%20origins.pdf. Consulta: 6 de noviembre de 2015. DOI: 10.1016/s10967494(01)00041-1.

FLORES, Zenyazen (2015), “62 millones no pueden comprar alimentos”, Sección Económica, El Financiero, 23 de febrero. En línea: http://www.elfinanciero.com.mx/economia/repunta-indice-de-la-tendencia-laboral-de-lapobreza-al-cierre-de-2014.html. Consulta: 20 de mayo de 2015.

GRUENING, G. (2001), "Origin and theoretical basis of New Public Management", en International Public Management Journal, [en línea], núm. 4, pp. 1-25. Consulta: 6 de noviembre de 2015. Disponible en: http://eclass.uoa.gr/ modules/document/file.php/PSPA108/4NPM\%20origins.pdf.

HABERMAS, Jurgen y John RAWLS (1996), Debate sobre el liberalismo político, Barcelona, Paidós.

HOOD, C. y JACKSON, M. (1991), Administrative Argument, Aldershot, UK: Dartmouth. DOI: 10.2307/1964094.

LeEUW, F. L. (1996), “Performance Auditing, New Public Management and Performance Improvement: Question And Answers", Accounting, Auditing \& Accountability Journal, vol. 9, núm. 2, pp. 92-102.

MACHINEA, J. (2009), “La crisis financiera internacional: su naturaleza y los desafíos de política económica” [en línea], en Revista CEPAL, núm. 97, pp. 33-58. Consulta: 16 de enero de 2016. Disponible en: http://www.cepal. org/publicaciones/xml/7/35847/rve97machinea.pdf.

Maldonado Trujillo, Claudia (2013), "Presupuesto por resultados en América Latina: El desafío de la información de capacidades”, en Robinson, MARC, Elaboración de presupuestos basados en resultados, Manual sobre la elaboración de presupuestos basados en resultados, Materiales de Capacitación Centros Regionales de Aprendizaje sobre Evaluación y Resultados, CLEAR. En Línea: http://www.clearla.cide.edu/ sites/default/files/Manual\%20sobre\%2ola\%2oelaboraci\%C3\%B3n\%20de\%2opresupuestos\%20basados\%20en\%20 resultados_CLEAR.pdf. Consulta: 20 de julio de 2016.

MATOS BAzÓ, Ruth (2005), "Enfoques de evaluación de programas sociales: Análisis comparativo”, Revista de Ciencias Sociales, vol. XI, núm. 2, mayo-agosto, 360-377.

MoRAles-RúAN, María del Carmen, ShAMAH-LeVy, Teresa, Mundo-RosAs, Verónica, Cuevas-NASU, Lucía, RomeroMARTíneZ, Martín, VilLALPANDo, Salvador, y RiverA-Dommarco, Juan Ángel (2013), “Programas de ayuda alimentaria en México, cobertura y focalización”, Salud Pública en México, vol. 55, suplemento 2: 199-205. En línea: http://www.scielo.org.mx/pdf/spm/v55s2/v55s2a17.pdf.

Montesinos JUlve, V. (1999), “El cambio de la cultura organizativa de las Administraciones Públicas y el presupuesto”, en I Encuentro de Avila sobre Gestión y Políticas Públicas: La Reinvención del Gobierno, Avila, de 25 al 29 de octubre.

MARIÑEZ (2013) “La gestión del nuevo servicio público” en XVIII Congreso Internacional del CLAD sobre la reforma del Estado y la Administración Pública, del 31 oct. al 1 nov., Montevideo. Disponible en: http://www. freddymarinez.com/docs/marinez.pdf. Consulta: 13 de noviembre de 2015.

NinA BALTAZAR, Esteban (2008), "Modelos de evaluación de política y programas sociales en Colombia”, Papel político, vol.13, núm. 2, julio-diciembre: 449-471.

Nozick, R. (1974), Anarchy, State, and Utopia, Nueva York, Basic Books. OECD (1992), Regulatory Reform, Privatization and Competition Policy, París, OCDE.

MoORE, Mark H. (1998), Gestión estratégica y creación de valor en el sector público. Barcelona: Paidós.

OLíAS DE LIMA, Blanca (2001), La nueva gestión pública, Madrid: Pearson. 
Ortegón, Edgar, Juan Francisco PACHeco y Adriana PRIETo (2005), Metodología del Marco Lógico para la planificación, el seguimiento y la evaluación de proyectos y programas. Chile: Serie, manuales, núm. 42, Instituto Latinoamericano y del Caribe de Planificación Económica y Social, ILPES, CEPAL.

Ostrom, V. (1973), The Intellectual Crisis in American Public Administration, Tuscaloosa, Alabama University Press.

PADRón, Carlos Eduardo (1995), "La Evaluación de programas sociales”, Revista Venezolana de Análisis de Coyuntura, núm. 1, julio-diciembre, 173-183. En línea: http://www.sicht.ucv.ve:8080/bvirtual/doc/analisis\%20 de\%20coyuntura/contenido/volumenes/1995/pag173.pdf. Consulta: 15 de mayo de 2015.

PÉREZ-JÁCOME FrISCIONE, Dionisio (2009), Presupuesto basado en resultados: Origen y aplicación en México. En línea: http://www.hacienda.gob.mx/EGRESOS/sitio_pbr/Documents/Pbr_Mex_02072012.pdf. Consulta: 1 de abril de 2015.

RAmos, José María y Alberto ViLlalobos (2012), “Un Gobierno por resultados y la nueva gestión pública en México", en RAMOS, José María y Tonatiuh GUILLEN LóPEZ (coordinadores), Gobernanza por resultados en México. Eficacia directiva, 2006-2012: Colegio de la frontera Norte.

SÁNCHEZ, J. (2001), Gestión Pública y Governance, Toluca: Instituto de Administración Pública del Estado de México.

SALCEDo, Aquino Roberto (2011), “Evaluación de políticas públicas”, en Evaluación de políticas públicas. México D. F.: núm. 13, compilador: Biblioteca Básica de Administración Pública y Siglo XXI Editores.

SECRETARIA DE DESARROLLO SOCIAL SEDESOL (2012), Diagnóstico integral de los programas alimentarios de SEDESOL, MD Consultoría. En línea: http://www.sedesol.gob.mx/work/models/SEDESOL/Sedesol/sppe/dgap/diagnostico_ alimentacion/Diagnostico_Alimentario_Version_Completa.pdf. Consulta: 15 de abril de 2015.

SECRETARIA DE DeSARROLlo SÖCIAL SEDESŌ (2013), Comedores Comunitarios. En línea: http://www.normateca. sedesol.gob. $\mathrm{mx} /$ work/models/NORMATECA/Normateca/1_Menu_Principal/2_Normas/2_Sustantivas/ Lineamientos_Prog_Comedores_Comunitarios.pdf. Consulta: $2 \overline{0}$ de abril de 2015.

SECRETARIA DE DESARROLLO SOCIAL SËDESOL, 2014, Elementos Técnicos de Diseño, Planeación e Instrumentación del Programa Nacional México Sin Hambre, julio 2014. En línea: http://www.sedesol.gob.mx/work/models/SEDESOL/ Cruzada/Programa_Nacional_Meexico_Sin_Hambre_Elementos_Metodologicos.pdf. Consulta: 6 de abril de 2015.

Secretaria de Desarrollo Social SEDESOl, b (2014), Diagnóstico y Propuesta de Atención de Programa de Comedores Comunitarios. En línea: http://www.sedesol.gob.mx/work/models/SEDESOL/Resource/2910/1/images/ Diagnostico_2014_PCC.pdf. Consulta: 17 de julio de 2015.

VICHER, D. (2007, septiembre), “La reforma neogerencial en Nueva Zelanda”, en Revista de Ciencias Sociales, vol. 14, núm. 45: 163-185.

VERA MARTínEZ, Martín (2015), Diseño y operación de indicadores de Evaluación en la AP, manual. 